\title{
Tumores testiculares. Evaluación de la experiencia durante 25 años en un hospital militar
}

\author{
R. Diz Rodríguez, M. Virseda Chamorro, P. Paños Lozano, J.A. Cabrera Cabrera, I. Arance Gil, \\ C. Alpuente Román
}

\author{
Servicio de Urología. Hospital Central de la Defensa. Madrid.
}

Actas Urol Esp 2005; 29 (5): 457-464

\section{RESUMEN}

TUMORES TESTICULARES. EVALUACIÓN DE LA EXPERIENCIA DURANTE 25 AÑOS EN UN HOSPITAL MILITAR

Objetivos: Las neoplasias testiculares son frecuentes en el adulto joven, coincidiendo con la incorporación al Servicio Militar. El objetivo de nuestro trabajo es evaluar las características tumores, su evolución con el tiempo y el resultado de los tratamientos empleados en nuestro centro para este tipo de tumores.

Material y métodos: Se realizó un estudio longitudinal retrospectivo en una cohorte de 98 pacientes de edad media 28,6 años, sometidos a orquiectomía por neoplasia testicular en nuestro centro entre los años 1979 a 2004 . En el estudio se recogieron los datos referentes a la edad de los pacientes, las características tumorales, el tratamiento realizado y la evolución de la neoplasia.

Resultados: En un 61\% de los casos, el testículo afectado fue el derecho (diferencias significativas). El tipo histológico más común fueron los tumores germinales no seminomatosos (TGNS) $(65,3 \%)$ de los casos seguidos de los seminomas puros $(27,6 \%)$, y de tumores no germinales (TNG) $(7,1 \%)$. Los TGNS se diagnosticaron a una edad media $(23,2$ años) significativamente menor que los otros dos tipos. El estadio I fue el más frecuente (58\%). Los seminomas presentaron un estadio I con una mayor frecuencia significativamente mayor (80\%) que el resto de los tumores. Los datos recogidos durante los 25 años no mostraron una variación significativa respecto a las características tumorales. El análisis de supervivencia indicó que las características tumorales con mejor pronóstico respecto a la probabilidad de recurrencias tumorales fueron los seminomas y los estadios tumorales I y II.

Conclusiones: Los tumores germinales no seminomatosos (TGNS) son los más frecuentes en adultos jóvenes. La mayoría de los tumores se diagnostican en estadios iniciales, y su pronóstico es más favorable en el caso de los seminomas y los estadios I y II.

Palabras clave: Neoplasia testicular. Factores pronósticos. Tratamiento. Análisis de supervivencia.

\section{ABSTRACT}

TESTICULAR NEOPLASMS. EVALUATION OF THE EXPERIENCE DURING 25 YEARS IN A MILITARY HOSPITAL

Objetives: The testicular tumors are frequent in the young adult, coinciding with the incorporation to the Military Service. The objective of our work is to evaluate the tumoral characteristic, its evolution with the time and the result of the treatments used in our center for this type of tumors.

Material and methods: We carried out a retrospective longitudinal study in a cohort of 98 patients with an average age of 28,6 years, subjected to orchiectomy for testicular tumor in our center between 1979 to 2004 . In the study, we collected data referred to the age of the patients, the tumoral characteristic, the outcome of the treatment and the evolution of the tumor.

Results: In $61 \%$ of the cases, the affected testicle was the right (significant differences). The most common histologyc type was the non seminomatous germ cell tumors (NSGCT) $(65,3 \%)$. Followed by the pure seminomas $(27,6 \%)$, and non germinal cell tumors (NGC) $(7,1 \%)$. The NSGCT was diagnosed to a average age $(23,2$ years) significantly smaller that the other two types. The stage I was the most frequent (58\%). The seminomas presented a stage I in a significantly bigger frequency (80\%) that the others tumors. The data picked up during the 25 years didn't show a significant variation regarding the tumoral characteristic. The survival análisis indicated that the tumoral characteristic with better pronostic regarding the probability of tumoral recurrences were the seminomas and the tumoral stages I and II.

Conclusions: The non seminomatous germ cell tumors (NSGCT) are the most frequent testicular tumors in young adults. Most of the tumors are diagnosed in initial stages, and their pronostic is better in the case of the seminomas and in the stages I and II.

Keywords: Testicular tumors. Pronostic factors. Treatment. Survical análisis. 
$\mathrm{L}$ os tumores testiculares representan alrededor del 1\% de los tumores del varón ${ }^{1}$. El 95\% de los tumores testiculares corresponde a tumores germinales cuya máxima incidencia tiene lugar entre la segunda y tercera década de la vida adulta ${ }^{2}$, periodo que corresponde a la etapa militar. Hasta la desaparición del Servicio Militar Obligatorio en 1999, la mayoría de los jóvenes dependian para su asistencia hospitalaria de la sanidad militar, por lo que el número de pacientes tratados con esta patología fue importante. Además al tratarse de un grupo homogéneo de pacientes es más interesante seguir su evolución a lo largo de un periodo de tiempo ${ }^{3}$.

El objeto de este trabajo es presentar las características tumorales, su evolución en el tiempo y el resultado de los tratamientos empleados en los pacientes tratados en nuestro centro por neoplasia testicular.

\section{MATERIAL Y MÉTODOS}

Se realizó un estudio retrospectivo sobre una cohorte de 98 pacientes sometidos a orquiectomía por neoplasia testicular entre enero de 1979 y julio de 2004. La edad media de los pacientes fue de 28,6 años (desviación típica 14,0 años).

En el estudio anatomo-patológico se registró el tamaño y localización del nódulo tumoral, así como la estirpe celular del tumor. El tipo histológico de tumor se determinó según la clasificación de Mostofi ${ }^{4,5}$. Para el diagnóstico de extensión se realizó una radiografía simple de tórax, y un TAC torácico y abdominal. El estadio tumoral se determinó de acuerdo con la clasificación del Royal Madsen Hospital $^{6}$.

Los datos de los pacientes fueron almacenados en una base de datos ACCESS y posteriormente importados al programa informático SPSS (versión 10), para su procesamiento estadístico. En el análisis estadístico se utilizó el test de la distribución binomial para contrastar la aleatoriedad de una variable dicotómica, el test de la chi-cuadrado de Pearson para comparar dos variables cualitativas, el test de comparación de medias de la t de Student para comparar dos variables cuantitativas, y el análisis de la variancia (ANOVA) con el test de contraste post hoc de Sheffe para comparar más de dos variables cuantitativas. El análisis de supervivencia se calculó según el método de Kaplan-Meier con el test Log-rank para comparar factores pronósticos. El nivel de significación se fijó en el 95\% bilateral.

\section{RESULTADOS}

1.- Características tumorales

El dato sobre la lateralidad del testículo afectado pudo ser recogido en 84 casos, correspondiendo 33 casos (39\%) al testículo izquierdo y 51 casos (61\%), al testículo derecho. Diferencias significativas respecto a la hipótesis de que la probabilidad para ambos testículos es la misma (distribución binomial $\mathrm{p}=0,012$ ). El tipo histológico de tumor se muestra en la Tabla 1. Los tumores
Tabla 1

Tipo histológico de neoplasia testicular

\begin{tabular}{lcc}
\hline Estirpe tumoral & No pacientes & Porcentaje \\
\hline Seminomas puros & & \\
Seminoma clásico & 23 & 23,5 \\
Seminoma anaplásico & 3 & 3,1 \\
Seminoma espermatocítico $\quad 1$ & 1,0 \\
$\quad$ Total seminomas & 27 & 27,6 \\
Otros tumores germinales puros & & \\
Carcinoma embrionario & 26 & 26,5 \\
Coriocarcinoma & 1 & 1,0 \\
Teratoma maduro & 5 & 5,1 \\
Teratoma inmaduro & 1 & 1,0 \\
$\quad$ Total otros tumores germinales puros & 33 & 33,6 \\
Seminoma mixto & & \\
Seminoma + Ca embrionario & 3 & 3,1 \\
Seminoma + teratoma & 2 & 2,0 \\
Seminoma + teratocarcinoma $\quad 7$ & 7,1 \\
$\quad$ Total seminomas mixtos & 12 & 12,2 \\
Otros tumores germinales mixtos & & \\
Teratocarcinoma & 16 & 16,3 \\
Ca embrionario + coriocarcinoma & 3 & 3,1 \\
$\quad$ Total otros tumores germinales mixtos & 19 & 19,4 \\
Tumores no germinales & & \\
Tumor de Leydig & 3 & 3,1 \\
Linfoma de alto grado & 2 & 2,0 \\
Sarcomas $\quad$ Total tumores no germinales & 2 & 2,0 \\
$\quad$ Total & 7 & 7,1 \\
$\quad 98$ & 100 \\
\hline$\quad$ & & \\
& &
\end{tabular}


germinales no seminomas fueron el tipo de tumor más frecuente $(65,2 \%)$. El estadio tumoral se muestra en la Tabla 2. El estadio I fue el más frecuente (58\%). La relación entre el estadio y la estirpe tumoral se muestra en la Tabla 3 . Se observó que los seminomas se presentaban con un estadio tumoral I en una proporción significativamente mayor que el resto de tumores testiculares.

Tabla 2

Estadio tumoral

\begin{tabular}{lccc}
\hline Estadio & $\begin{array}{c}\text { No de } \\
\text { pacientes }\end{array}$ & $\begin{array}{c}\text { Porcentaje } \\
\text { total }\end{array}$ & $\begin{array}{c}\text { Porcentaje } \\
\text { válido }\end{array}$ \\
\hline Estadio I & 34 & 34,7 & 58 \\
Estadio II & 18 & 18,4 & 31 \\
Estadio III & 5 & 5,1 & 8 \\
Estadio IV & 2 & 2,0 & 3 \\
Total & 59 & 60,2 & 100 \\
Valores perdidos & 39 & 39,8 & \\
Total & 98 & 100 & \\
\hline
\end{tabular}

Tabla 3

Relación entre el estadio y la estirpe histológica tumoral

\begin{tabular}{lccc}
\hline Estadio & $\begin{array}{c}\text { Estadio I } \\
\text { No }\end{array}$ & $\begin{array}{c}\text { Superior } \\
\text { al Estadio I } \\
\text { № }\end{array}$ & $\begin{array}{c}\text { Total } \\
\text { Noo }\end{array}$ \\
\hline $\begin{array}{l}\text { Seminoma puro } \\
\text { Otros tumores }\end{array}$ & $16(80 \%)$ & $4(20 \%)$ & $20(100 \%)$ \\
testiculares & $20(51 \%)$ & $19(49 \%)$ & $39(100 \%)$ \\
Total & $36(61 \%)$ & $23(39 \%)$ & $59(100 \%)$ \\
\hline
\end{tabular}

Prueba de la chi-cuadrado de Pearson p=0,030 (significativo).

El tamaño medio del nódulo tumoral fue de de $5,17 \mathrm{~cm}$ (desviación típica: $3,22 \mathrm{~cm}$ ). No se encontraron diferencias significativas en el tamaño del tumor entre los diferentes tipos histológicos de neoplasia testicular, ni entre los diferentes estadios tumorales

Respecto a la edad, se observó una diferencia significativa entre los tumores germinales no seminomatosos y los otros dos grupos (seminomas y tumores no germinales). Aunque no entre estos dos grupos entre sí (ANOVA, $\mathrm{p}=0,000$ y test post hoc de Sheffe) (Fig. 1).

2.- Evolución de las características tumorales

La proporción del tipo histológico de tumor y el estadio tumoral en cada uno de los cinco lustros de estudio se muestran en las Figuras 2 y 3.

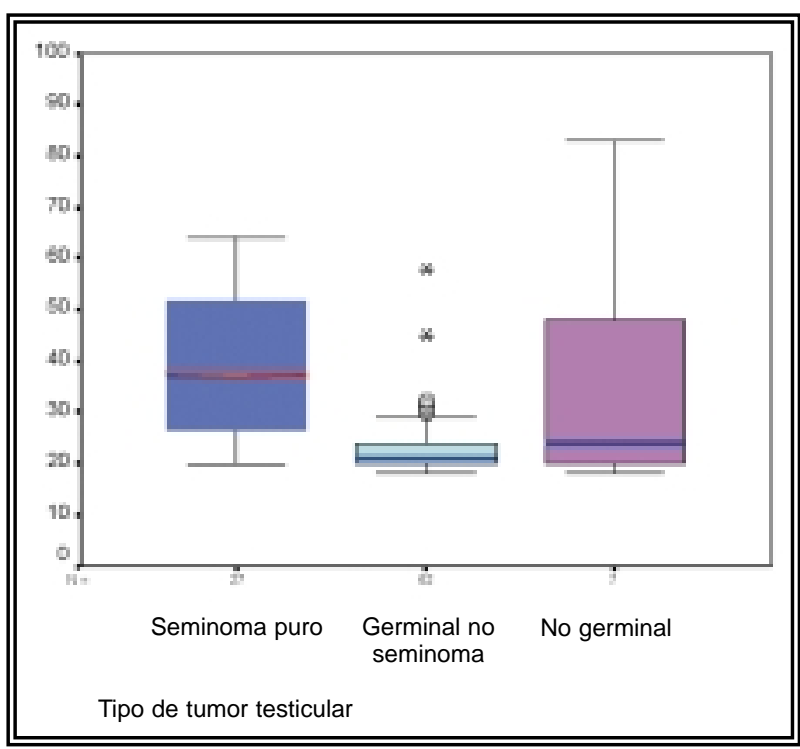

FIGURA 1. Relación entre la edad media y la estirpe tumoral. Diagrama de caja (box-plot) que representa la distribución de edades de los tres grupos de tumores vesicales. La media de edad de los tumores germinales no seminomatosos fue de 23,2 años (desviación tipica: 6,5 años), la media de edad de los seminomas fue de 39,3 años (desviación típica: 14,9 años) y la de los tumores no germinales 37, 3 años (desviación típica: 27,5 años).

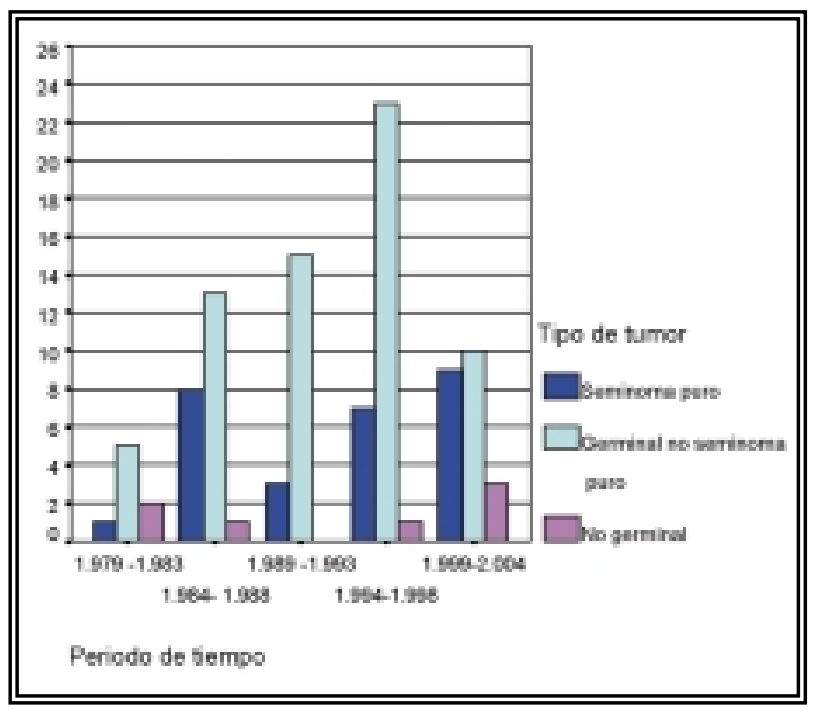

FIGURA 2. Evolución del tipo de tumor durante el periodo de estudio.

No se observaron diferencias significativas respecto a estos parámetros en las distintas fechas diagnósticas.

\section{3.- Seguimiento y resultados terapéuticos}

La mediana del tiempo de seguimiento fue de 45,73 meses (amplitud intercuartil: 87,43 meses). En 9 casos hubo progresión tumoral, correspon- 


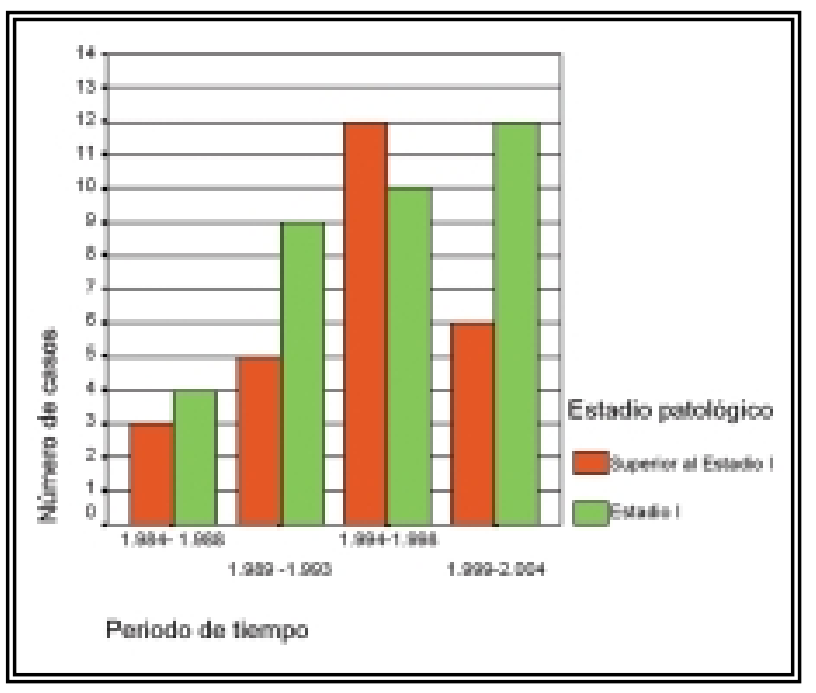

FIGURA 3. Evolución del estadio tumoral durante el periodo de estudio.

diendo a recidiva en el otro testículo en cuatro casos, recidiva retroperitoneal en 3 casos y fallecimiento en 2 casos.

El seguimiento fue lo suficientemente prolongado para poder evaluar el resultado del tratamiento en 58 casos. El tratamiento efectuado en estos pacientes, según el tipo histológico y el estadio tumoral, así como el número de los que progresaron en cada una de las categorías se recoge en la Tabla 4.
El análisis de supervivencia según la estirpe tumoral y el estadio se muestran en las Figuras 4 y 5. El test de Log-rank mostró una diferencia significativa en cuanto a la supervivencia según la estirpe tumoral $(\mathrm{p}=0,000)$ y el estadio $(\mathrm{p}=0,000)$, excepto entre los estadios I y II, entre los que no hubo diferencias significativas respecto a la supervivencia.

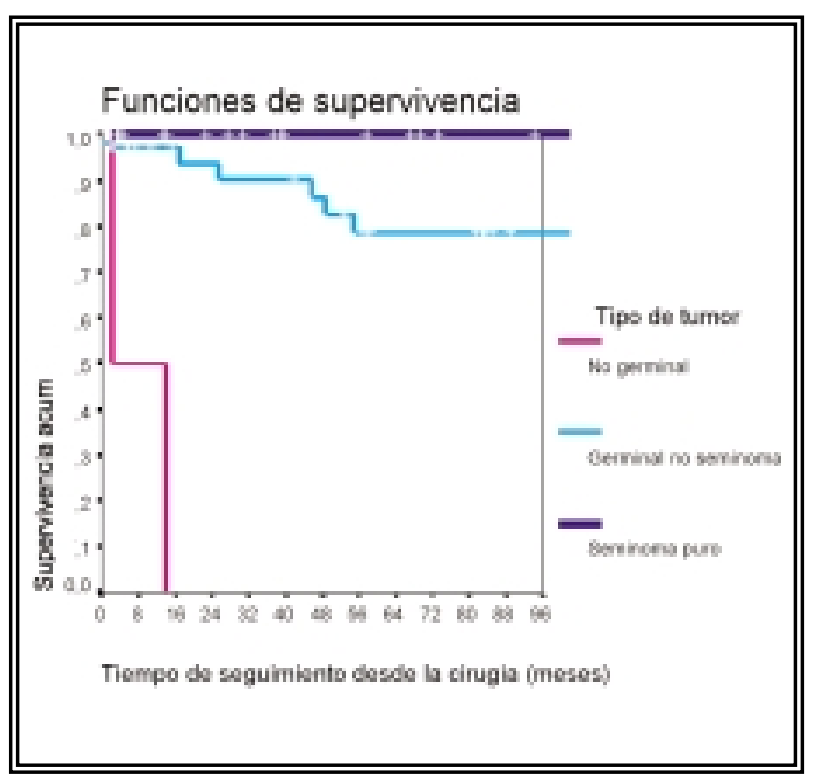

FIGURA 4. Análisis de supervivencia en función de la estirpe tumoral.

Tabla 4

Relación entre el estadio y tipo tumoral el tratamiento complementario y la progresión tumoral

\begin{tabular}{|c|c|c|c|c|c|c|c|c|}
\hline & & ORQ & LFD & RDT & QMT & L+QMT & $\mathrm{R}+\mathrm{QMT}$ & Total \\
\hline \multirow[t]{3}{*}{ Estadio I } & SEM & (1) 10 & 0 & 3 & 3 & 0 & 0 & (1) 16 \\
\hline & GNS & (2) 9 & 4 & 0 & 5 & 0 & 0 & (2) 18 \\
\hline & NG & (1) 1 & 0 & 0 & 0 & 0 & 0 & (1) 1 \\
\hline \multirow[t]{2}{*}{ Estadio II } & SEM & 0 & 0 & 1 & 2 & 0 & 0 & 3 \\
\hline & GNS & 0 & 0 & 1 & (2) 7 & 6 & 0 & (2) 14 \\
\hline \multirow[t]{3}{*}{ Estadio III } & SEM & 0 & 0 & 0 & 1 & 0 & 0 & 1 \\
\hline & GNS & 0 & 0 & 0 & (1) 3 & 0 & 0 & (1) 3 \\
\hline & $\mathrm{NG}$ & 0 & 0 & 0 & 0 & 0 & (1) 1 & (1) 1 \\
\hline \multirow[t]{2}{*}{ Estadio IV } & GNS & 0 & 0 & 0 & (1) 1 & 0 & 0 & (1) 1 \\
\hline & Total & (4) 20 & 4 & 5 & (4) 22 & 6 & (1) 1 & (9) 58 \\
\hline
\end{tabular}

ORQ: sólo orquiectomía. LFD: linfadenectomía. RDT: radioterápia. QMT: quimioterapia, L+QMT: linfadenectomía y quimioterpia, R+QMT: radioterapia y quimioterapia. Entre paréntesis número de casos de cada una de las categoría con progresión tumoral. 


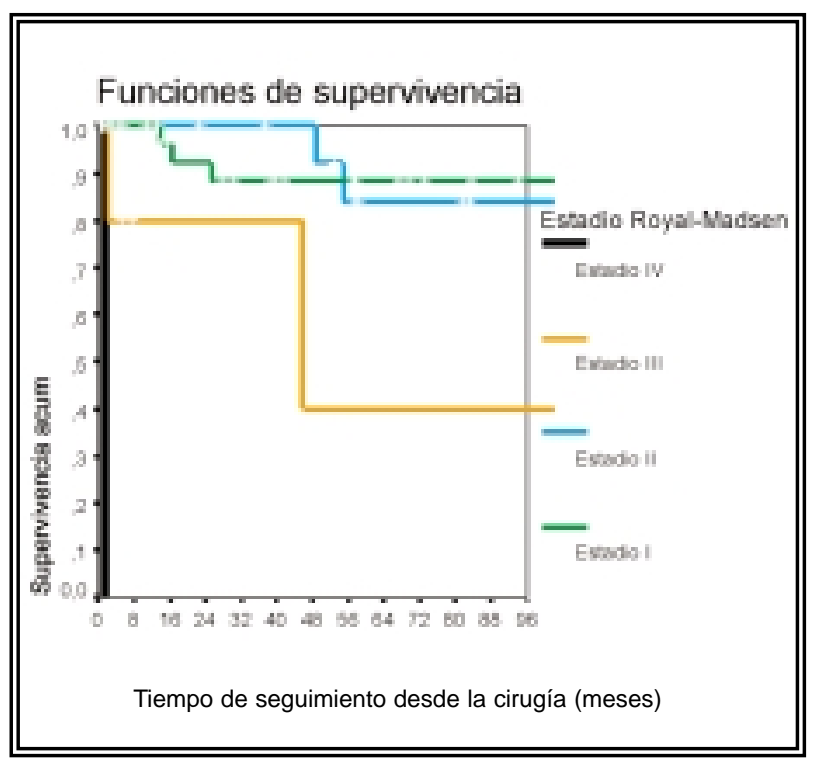

FIGURA 5. Análisis de supervivencia en función del estadio tumoral.

\section{DISCUSION}

En nuestra serie se comprobó una mayor afectación tumoral en el testículo derecho. Este dato coincide con lo expuesto en otras series ${ }^{7,8}$. Algunos autores consideran que los tumores germinales son más frecuentes en el testículo derecho, mientras que sarcomas y los tumores de personas mayores predominan en el testículo izquierdo ${ }^{9}$.

Los tumores germinales no seminomatosos (TGNS) fueron el tipo histológico de tumor más frecuente $(65,2 \%)$, coincidiendo con otras series españolas ${ }^{7,10,11}$. Aunque en otros países occidentales la proporción se invierte ligeramente ${ }^{12,13}$, la distribución de los tipos de tumor suele ser semejante, y contrasta abiertamente con la distribución en otros países, como los africanos, en los que predominan los linfomas ${ }^{14}$. La prevalencia del tipo de tumor no parece estar influenciado por factores genéticos (como la raza) ${ }^{15}$. Por el contrario, parece que los factores ambientales son los más importantes ${ }^{16}$.

En nuestro estudio no se observó un cambio significativo respecto a la proporción del tipo de neoplasia testicular a lo largo del tiempo. En los cinco lustros estudiados los TGNS (tumores germinales no seminomatosos), fueron más frecuentes que los seminomas puros. Si bien es cierto, que en el último lustro, las frecuencias de ambos tipos de tumores han ido aproximándose. Un resultado semejante ha sido referido por otros estudios españoles ${ }^{7,17}$. Algunos autores europeos han notado un incremento en la incidencia en todos los tipos de neoplasia testiculares (seminoma y TGNS) $^{18}$, mientras que para otros autores serían únicamente los seminomas, los que aumentarian su incidencia, especialmente en la década de los noventa del pasado siglo ${ }^{19}$. Por último, para algunos autores americanos los seminomas aumentarían su incidencia únicamente en la población blanca, permaneciendo estable en la población negra ${ }^{20}$. Estas conclusiones son contrarias a la supuesta independencia de la raza sobre el tipo de neoplasia testicular ${ }^{15}$. En nuestro estudio también se observó un incremento constante del número de neoplasias testiculares registradas hasta el año 1999. Esto se explica teniendo en cuenta que hasta esa fecha el Servicio Militar era obligatorio, por lo que el contingente de la población atendida permanecía estable. Al suprimirse la obligatoriedad del Servicio Militar, nuestra población de referencia disminuyó apreciablemente, originando un descenso del número de neoplasias testiculares tratadas. En definitiva, aunque el aumento de incidencia de las neoplasias tumorales parece una cuestión evidente, el aumento de la incidencia de seminomas está todavía por dilucidar.

El estadio tumoral más frecuente en nuestra serie fue el estadio I (58\% de los casos). En este parámetro también coincidimos con otras series españolas ${ }^{7,10,11}$ y de otros países occidenta$\operatorname{les}^{8,15}$. También se observó un estadio patológico menos avanzado en los seminomas respecto a los otros tipos de tumores testiculares: un $80 \%$ de estadios I en los seminomas, frente a un $51 \%$ en el resto de los tumores. Estas proporciones son semejantes a las referidas en otras series $^{7,11,17,18}$.

El estadio tumoral más frecuente fue el estadio I en tres de los cuatro lustros estudiados. Únicamente en el lustro de 1.994 a 1.999 se invirtió esa proporción. Otros autores como Hernes et al. ${ }^{18}$ observan un aumento del porcentaje de estadios iniciales (estadio I) con el tiempo, pasando del $57 \%$ al $72 \%$ en doce años. Estos autores atribuyen este cambio a la mejora en el 
diagnóstico de la neoplasia testicular. En nuestra cohorte también observamos un aumento del porcentaje de pacientes diagnosticados en el estadio inicial de la enfermedad, excepto en la cohorte de pacientes diagnosticados entre 1994 y 1998. Esta anomalía puede deberse al aumento de la proporción de TGNS (un 23\%), y a un aumento del tiempo transcurrido entre el primer síntoma y la cirugía, debido a que en este periodo la presión asistencia fue máxima. El intervalo entre el primer síntoma y la cirugía ha demostrado su influencia sobre el estadio tumoral en el momento del diagnóstico ${ }^{7,18}$.

En nuestra serie no se comprobó ninguna relación entre el tamaño del tumor testicular y sus características histológicas o evolutivas. Vergouwe et al. ${ }^{21}$ en un metaananálisis sobre un total de 2.587 pacientes, encuentran sólo una débil asociación entre el tamaño tumoral y la progresión en el caso de tumores testiculares no seminomatosos.

La edad si mostró una relación significativa con la estirpe tumoral, siendo la edad media de los pacientes con TGNS significativamente menor que la de los otros dos grupos de tumores. La media de edad de los seminomas fue de 39,2 años y la de los TGNS 23, 2 años. Estas diferencias también se observan en otras series tanto nacionales ${ }^{7,17}$, como extranjeras ${ }^{8,18}$. Respecto a los tumores no germinales, en nuestra serie se incluyen tres tipos histológicos diferentes: los sarcomas, que serían más frecuentes en población joven ${ }^{22}$ (en nuestra serie los dos casos fueron menores de 18 años), los tumores de Leydig que correspondería a una edad más avanzada $^{23}$ (en nuestra serie sus edades estuvieron comprendidas entre los 21 y los 25 años), y por último los linfomas que serían típicos de edades avanzadas ${ }^{10,24}$ (en nuestra serie ambos fueron ocurrieron en personas mayores de 70 años).

Existe una relación evidente entre el tipo histológico y el estadio tumoral y el pronóstico de esta neoplasia. El análisis de la supervivencia mostró en nuestra cohorte de pacientes que tanto el padecer un seminoma, como ser diagnosticado en estadios iniciales de la enfermedad (estadios I y II) son factores de buen pronóstico respecto a la progresión del tumor. El tratamiento de nuestros pacientes se ajustó a este supuesto. Así, en los 10 casos de seminomas en estadio I que se trataron únicamente con orquiectomía sólo se produjo una progresión tumoral (que tuvo lugar a los 12 años), mientras que en ninguno de los casos de seminoma tratados con quimioterapia o radioterapia se produjeron progresiones. En otras series, el porcentaje de progresiones en seminomas con estadio I varía entre el $16 \%$ sin tratamiento, el $11 \%$ con radioterapia y el $3 \%$ con quimioterapia ${ }^{7}$. Ante estos datos está en discusión la necesidad de añadir otro tratamiento a este tipo de pacientes. Algunos autores postulan la necesidad de tratar a todos los seminomas en estadio inicial con radioterapia en los campos paraaórtico con el fin de prevenir recidivas retroperitoneales ${ }^{17,25}$. Sin embargo, no siempre es necesario un tratamiento complementario. Warde et al. ${ }^{26}$ consideran que el riesgo de recidivas retroperitoneales es sólo del $6 \%$ a los 5 años de seguimiento en pacientes mayores de 34 años, con tumor menor de $6 \mathrm{~cm}$ y sin invasión tumoral. En estos casos estaría indicada únicamente la vigilancia ${ }^{27}$. La quimioprofilaxis en este tipo de tumores con carboplatino también ha sido utilizada por algunos autores ${ }^{28}$. Sin embargo, todavía no existen estudios a largo plazo sobre la eficacia de este tratamiento $^{27}$.

Los tumores germinales no seminomatosos (TGNS) en el estadio inicial se trataron en nuestra serie únicamente con orquiectomía y vigilancia en 9 casos, se asoció linfadenectomía retroperitoneal en 4 casos y poliquimioterapia en 5 casos. De los 8 casos sin tratamiento complementario hubo 2 recidivas retroperitoneales, por ninguna en los casos con linfadenectomía y poliquimioterapia. En la serie de Germá-Lluch, et al. ${ }^{7}$ hubo un $22 \%$ de recidivas en pacientes sometidos únicamente a vigilancia frente a un $2 \%$ en el caso de los pacientes que recibieron poliquimioterapia. La linfadenectomía en este tipo de tumor pone en evidencia que alrededor de un $30 \%$ de los pacientes están infraestadiados (tienen metástasis linfáticas retroperitoneales). En este caso es preciso realizar quimioterapia complementaria con bleomicina, etopóxido y cisplatino (BEP). En el caso de que los nódulos sean negativos, la probabilidad de recidivas continúa siendo del $10 \% 27$. Se considera que los factores de riesgo de progre- 
sión tumoral son: la presencia de invasión vascular, y la positividad del anticuerpo monoclonal MIB-1 (signo de actividad proliferativa), y en menor medida la edad y el tamaño del tumor. En pacientes sin factores de riesgo la vigilancia es igual de efectiva que el tratamiento complementario $^{21}$. En caso de pacientes con factores de riesgo estaría indicado tanto la linfadenectomía como la poliquimioterapia con BEP. Al considerarse que la quimioterapia es mejor tolerada que la linfadenectomía, este último tratamiento ha sido progresivamente abandonado ${ }^{29}$.

En los tumores con estadio II, se considera que el tratamiento inicial debe ser la quimioterapia mediante $\mathrm{BEP}$ o $\mathrm{PEB}$ (cisplatino, etopóxido y bleomicina). En nuestra serie ningún paciente con seminoma presentó recidivas, mientras que entre los pacientes con TGNS hubo dos recidivas $^{27}$. Germá-Lluch, et al. ${ }^{7}$ refieren un $9 \%$ de progresiones con este tratamiento. La alternativa es la radioterapia para los seminomas ${ }^{30}$, utilizada en nuestra serie en un caso, o la linfadenectomía para los TGNS, empleada junto a la quimioterapia en seis de nuestros pacientes, todas ellas con buenos resultados. En cualquier caso es fundamental remarcar la importancia que supuso la introducción a finales de la década de los setenta del pasado siglo de la quimioterapia basada en el cisplatino, que generó un cambio radical en el pronóstico de este tipo de tumores. Esto supuso por ejemplo en Islandia un incremento de la supervivencia a los 5 años del 67\% en el periodo de 1955 a 1977, al 96\% en el periodo de 1978 a $1999^{12}$. Por desgracia, en algunos países africanos que aún no disponen de este fármaco, la mortalidad continúa siendo elevada $^{31}$.

Los estadios III y IV, constituyen factores de mal pronóstico. En estos casos se suele utilizar quimioterapia mas agresiva como PEB o PEI (cisplatino, etopóxido e ifosfamida), aunque no está claro que aumenten la tasa de superviven$\operatorname{cia}^{27}$.

Por último, los tumores no germinales también tienen un peor pronóstico, bien debido a que son más agresivos o se presentan en personas de edad avanzada como los linfomas ${ }^{24}$, o bien a que tiene tendencia a la bilateralidad como los tumores de Leydig 32 .

\section{REFERENCIAS}

1. Schottenfield D, Warshauwe ME, Sherlock S, Zauber AG, Leyder M, Payne R: The epidemiology of testicular cancer in young adults. Am J Epidemiol 1980;112:232-246.

2. Richie JP. Neoplasm of the testis. In: Campbell's Urology $7^{\text {th }}$ ed. Walsh PC et al, (edts): p.2411-2452, Wb Saunder Co: Philadelphia, 1997.

3. Yamane GK, Johnson R. Testicular carcinoma in U.S. Air Force aviators: a case-control study. Aviat Space Environ Med 2003;74(8):846-850.

4. Mostofi FK, Sobin LH. International histological clasification of tumor of the teste. WHO: Geneve, 1977.

5. Mostofi FK, Sesterhenn IA. Histological typing of the testis tumors. WHO International Histological Clasification Tumors $2^{\text {nd }}$ Edition. Springer-Verlag: Berlin, 1998.

6. Medical Research Council Working Party on Testiculars Tumours. Prognostic factors in advanced non-seminomatous germ-cell testicular tumours: results of multicentre study. Lancet 1985;1:8-11.

7. Germa-Lluch JR, García del Muro X, Maroto P, et al. Spanish Germ-Cell Cancer Group (GG). Clinical pattern and therapeutic results achieved in 1490 patients with germ-cell tumours of the testis: the experience of the Spanish Germ-Cell Cancer Group (GG). Eur Urol 2002; 42(6):553-563.

8. Tomomasa H, Shimizu H, Sato S, et al. Clinical study of testicular germ cell tumors. Hinyokika Kiyo. 2001 Jun;47(6): 389-395.

9. Stone JM, Cruickshank DG, Sandeman TF, Matthews JP Laterality, maldescent, trauma and other clinical factors in the epidemiology of testis cancer in Victoria, Australia. $\mathrm{Br}$ J Cancer.1991Jul;64(1):132-138.

10. Soto delgado M, Varo C, Juárez A, Bachiller J, Beltrán V, González D, Sánchez bernal C. Consideraciones sobre tumores de testículo: nuestra experiencia. Arch Esp Urol 1999; 52(2):123-132.

11. Fernández Gómez JM, Guate Ortiz JL, Martín Huescar A, et al. Presentación clínica del cáncer germinal de testículo. Arch Esp Urol 2002;55(8):915-922.

12. Gudbjartsson T, Magnusson K, Bergthorsson J, et al. A population-based analysis of increased incidence and improved survival of testicular cancer patients in Iceland. Scand J Urol Nephrol 2003;37(4):292-308.

13. Abratt RP, Reddi VB, Sarembock LA. Testicular cancer and cryptorchidism Br J Urol 1992;70(6):656-659.

14. Angwafo FF 3rd, Takongmo S, Mbakop A, Ngu VA. Testes tumors in a Sub-Saharan African city (Yaounde). Incident cases and histopathology. Eur Urol 1996;30(3):345-348.

15. Bridges PJ, Sharifi R, Razzaq A, Guinan P. Decreased survival of black Americans with testicular cancer. J Urol 1998Apr; 159(4): 1221-1223.

16. Huyghe E, Matsuda T, Thonneau P. Increasing incidence of testicular cancer worldwide: a review. J Urol 2003 Jul; 170 (1):5-11.

17. Armora Mani J, Riera Canals L, Franco Miranda E, et al. Tumores germinales de testículo, casuística y revisión bibliográfica. Actas Urol Esp 1990Sep-Oct;14(5):319-324.

18. Hernes EH, Harstad K, Fossa. Changing incidence and delay of testicular cancer in southern Norway (1981-1992). Eur Urol 1996;30(3):349-357.

19. Levi F, Te VC, Randimbison L, La Vecchia C. Trends in testicular cancer incidence in Vaud, Switzerland. Eur J Cancer Prev 2003;Aug12(4):347-349. 
20. McGlynn KA, Devesa SS, Sigurdson AJ, Brown LM, Tsao L, Tarone RE. Trends in the incidence of testicular germ cell tumors in the United States. Cancer 2003Jan1;97(1):63 70 .

21. Vergouwe Y, Steyerberg EW, Eijkemans MJ, Albers P, Habbema JD. Predictors of occult metastasis in clinical stage I nonseminoma: a systematic review J Clin Oncol 2003;Nov15,21(22):4092-4099.

22. Erbay ME, Tarhan F, Barisik NO, Kuyumcuoglu U. A case of testicular rhabdomyosarcoma. Int Urol Nephrol 2004; 36(1):73-75

23. Cruceyra Betriu G, Tejido Sánchez A, Duarte Ojeda JM. et al. Tumor de Leydig: presentación de 8 casos y revisión de la literatura. Actas Urol Esp 2002Jan;26(1):36-40.

24. Post PN, Casparie MK, Ten Kate FJ, Oosterhuis JW. The epidemiology of tumors of the testes in the Netherlands: accurate rendering by the Registry of Histopathology and Cytopathology (PALGA) Ned Tijdschr Geneeskd 2004;148 (23): 1150-1154.

25. Antoni R, Barbera F, Bertoni F, et al. Stage I seminoma of the testis: a bi-institutional retrospective analysis of patients treated with radiation therapy only. BJU Int $2003 \mathrm{Jul} ; 92$ (1):47-52.

26. Warde P, Gospodarowicz MK, Banerjee D, et al. Prognostic factors for relapse in stage I testicular seminoma treated with surveillance. J Urol 1997;157(5):1705-1709.
27. Laguna MP, Klepp O, Horwich A, et al. Guidelines on testicular cancer. European Association of Urology, 2004.

28. Krege S, Kalund G, Otto T, Goepel M, Rubben H. Phase II study: adjuvant single-agent carboplatin therapy for clinical stage I seminoma. Eur Urol 1997;31(4):405-407.

29. Bohlen D, Borner M, Sonntag RW, Fey MF, Studer UE. Long-term results following adjuvant chemotherapy in patients with clinical stage I testicular nonseminomatous malignant germ cell tumors with high risk factors. J Urol 1999 Apr;161(4):1148-1152.

30. Classen J, Schmidberger H, Meisner C. et al. Radiotherapy for stages IIA/B testicular seminoma: final report of a prospective multicenter clinical trial. J Clin Oncol. $2003 \mathrm{Mar}$ 15;21(6):1101-1106.

31. Opot EN, Magoha GA. Testicular cancer at Kenyatta National Hospital, Nairobi. East Afr Med J 2000 Feb;77(2): 80-85.

32. Kim I, Young RH, Scully RE: Leydig cell tumor of the testis. A cliniccopathological analysis of 40 cases and review of the literature. Am J Sug Pathol 1985;9(3):177-192.

Dr. M. Vírseda Chamorro

C/ Argentina 4; $1^{\circ}-1$ a

28027 Madrid

(Trabajo recibido el 29 de octubre 2004) 